\title{
Cochannel Interference Computation for Arbitrary Nakagami Fading
}

\author{
C. Tellambura
}

\begin{abstract}
A characteristic function method is used to calculate the probability that the signal-to-interference ratio (SIR) drops below some predefined threshold (probability of outage). The mobile unit receives a desired signal which has undergone Nakagami fading and multiple, cochannel, and independent Nakagami interferers. The development is based on a technique due to Bealieu. The advantages of the method are twofold. First, it places no integral restriction upon the Nakagami fading parameter. Second, it avoids the evaluation of higher order derivatives (residues).
\end{abstract}

Index Terms - Cellular radio system, characteristic function, cochannel interference, Nakagami fading, outage.

\section{INTRODUCTION}

$\mathbf{I}$ $\mathrm{N}$ CELLULAR radio, highly efficient use of available spectrum is achieved by frequency reuse, which results in cochannel interference. Furthermore, the desired signal and interfering signals are subject to fading, and the probability of outage is a useful statistical measure of performance [1]. The effect of cochannel interference on system performance has recently been studied extensively (see [2] for many references). The Nakagami fading model [3] is sufficiently general to be useful in different fading environments.

Consider the evaluation of the probability of outage $P_{\text {out }}$ in a Nakagami fading environment. Let the short-term signal powers be given by independent random variables (RV's) $p_{k}$, $k=0$ denoting the desired signal, and $k=1, \cdots, L$ denoting the interfering signals. The probability density function (pdf) of $p_{k}$ is completely characterized by two parameters: the mean signal power $\bar{p}_{k}$ and the fading severity index $0.5 \leq m_{k}<\infty$. The one-sided Gaussian distribution $\left(m_{k}=0.5\right)$, Rayleigh distribution $\left(m_{k}=1\right)$, and nonfading case $\left(m_{k} \rightarrow \infty\right)$ are the special cases. The pdf of $p_{k}$ is the well-known Gamma distribution, and it easily follows that the moment generating function (MGF) is given by

$$
\phi_{p_{k}}(s)=E\left(e^{s p_{k}}\right)=\frac{1}{\left(1-s \bar{p}_{k} / m_{k}\right)_{k}^{M}}, \quad k=0,1, \cdots, L
$$

where $\operatorname{Re}(s)<m_{k} / \bar{p}_{k}$ defines the convergence strip for $\phi_{p_{k}}(s)$ in the complex plane.

The object of this paper is to compute the probability

$$
\operatorname{Pr}\left[q\left(p_{1}+\cdots+p_{L}\right)>p_{0}\right]
$$

Manuscript received February 14, 1996; revised March 24, 1997.

The author is with the School of Computer Science and Software Engineering, Monash University, Clayton, Victoria 3168, Australia.

Publisher Item Identifier S 0018-9545(99)01030-0. where $q$ is the power protection ratio. The power protection ratio, usually specified in decibels, is fixed by the type of modulation and transmission technique employed and the quality of service desired: $9<q<20$ (decibels).

Given the random variable

$$
\gamma=\left(\sum_{k=1}^{L} p_{k}\right)-p_{0} / q
$$

it follows that the outage is given as

$$
P_{\text {out }}=\operatorname{Pr}(\gamma>0) \text {. }
$$

The MGF $E\left(e^{s \gamma}\right)$ is given by

$$
\phi_{\gamma}(s)=\frac{1}{\left(1+s \bar{p}_{0} / q m_{0}\right)^{m_{0}}} \prod_{k=1}^{L} \frac{1}{\left(1-s \bar{p}_{k} / m_{k}\right)^{m_{k}}}
$$

where $-q m_{0} / \bar{p}_{0}<\operatorname{Re}(s)<\min \left\{m_{k} / \bar{p}_{k}: k=1, \cdots, L\right\}$ defines the convergence strip in the complex plane.

At least in principle, the pdf $f_{\gamma}(x)$ can be determined explicitly when all the $m_{k}$ 's are integers, using the usual inversion methods for the Laplace transform. That is, the pdf is expressed as a contour integral, with the vertical contour lying in the strip of convergence, and then evaluated via the residue theorem.

Abu-Dayya and Beaulieu [2] obtain an expression for $P_{\text {out }}$ using a method similar to the above. In particular, the pdf of the total interference is an expression involving higher order derivatives (i.e., residues). Therefore, to apply this method, the $m_{k}$ 's are constrained to take integer values. For nonintegral $m$, [2] suggests evaluating the probability of outage for $\lfloor m\rfloor$ and $\lceil m\rceil$ and linearly interpolating to get an approximate value for the outage.

From (2), one finds that the pdf $f_{\gamma}(x)$ for $-\infty<x<0$ is sufficient to determine $P_{\text {out }}$. Thus, when inverting $\phi_{\gamma}(s)$ to obtain $f_{\gamma}(x)$ in the desired range, only the residue at the pole $s=-q m_{0} / \bar{p}_{0}$ needs to be evaluated and only $m_{0}$ needs to be an integer. This is the gist of the method given by Zhang [4]. However, it still calls for an $\left(m_{0}-1\right)$ th-order derivative.

As the right-hand side of (2) relates to the complementary distribution (CDF) of $\gamma, P_{\text {out }}$ can be determined using an infinite series derived by Bealieu [5] for the CDF of a sum of independent random variables. This method completely avoids any derivatives, and, as will be shown, the errors involved can be controlled. 


\section{Outage Probability AND ERROR BoundS}

Using the series expression for the CDF [5, (6b)]

$$
P_{\text {out }}=\frac{1}{2}+\sum_{n=0}^{\infty} \operatorname{Im}\left\{\frac{\phi_{\gamma}[j(2 n+1) h]}{\pi(n+1 / 2)}\right\}
$$

where $j=\sqrt{-1}$ and $h$ is a parameter controlling the accuracy

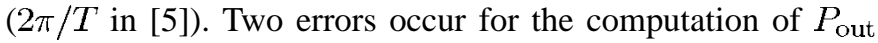
via this series expression. First, since this series is exact only for a sum of bounded RV's, a domain truncation error exists for a sum of unbounded RV's. Second, since the infinite series must be terminated, the remainder term injects an error. These errors will now be addressed.

The domain truncation error $\Delta$ can be bounded as [5, eq. (24)]

$$
|\Delta| \leq \max \{\operatorname{Pr}(\gamma \geq \pi / h), \operatorname{Pr}(\gamma<-\pi / h)\} .
$$

By applying the Chernoff bound to each term, we have

$$
\begin{aligned}
|\Delta| \leq \max \left\{\min _{\lambda>0} \phi_{\gamma}(\lambda) \exp \left(\frac{-\pi \lambda}{h}\right), \min _{\lambda<0} \phi_{\gamma}(\lambda)\right. \\
\left.\cdot \exp \left(\frac{\pi \lambda}{h}\right)\right\}
\end{aligned}
$$

where each minimization is to be done within the convergence strip of $\phi_{\gamma}(s)$ and on the real axis (i.e., $\lambda$ is real). It is not necessary to minimize with great precision. Let $\lambda_{1}=$ $-m_{0} q / 2 \bar{p}_{0}$ and $\lambda_{2}=\min \left\{m_{k} / 2 \bar{p}_{k}: k=1, \cdots, L\right\}$. Then

$$
|\Delta| \leq \max \left\{\phi_{\gamma}\left(\lambda_{2}\right) \exp \left(\frac{-\pi \lambda_{2}}{h}\right), \phi_{\gamma}\left(\lambda_{1}\right) \exp \left(\frac{\pi \lambda_{1}}{h}\right)\right\}
$$

Assume the series (4) is terminated at $n=N$ (i.e., the terms $n>N$ are neglected). Then, the remainder term $R_{N}$ is bounded as

$$
\begin{aligned}
\mid R_{N} & \leq \int_{u=N}^{\infty} \frac{\left|\phi_{\gamma}(j(2 u+1) h)\right|}{\pi(u+1 / 2)} d u \\
& \leq \frac{1}{K_{2}\{(N+1 / 2) h\}^{K_{1}}}
\end{aligned}
$$

where $K_{1}=m_{0}+m_{1}+\cdots+m_{L}$ and

$$
K_{2}=\pi K_{1}\left(\frac{2 \bar{p}_{0}}{q m_{0}}\right)^{m_{0}} \prod_{k=1}^{L}\left(\frac{w \bar{p}_{k}}{m_{k}}\right)^{m_{k}} .
$$

\section{EXAMPLES}

Let the required accuracy be $\epsilon$. Now, the steps for the computation of $P_{\text {out }}$ are as follows.

\begin{tabular}{|c|c|c|c|c|c|c|c|c|}
\hline \multirow{2}{*}{$\operatorname{SIR}(\mathrm{AB})$} & \multicolumn{4}{|c|}{$7 m_{0}=1$} & \multicolumn{4}{|c|}{$m_{0}=1.5$} \\
\hline & $h$ & $N$ & Series & Fxact & $h$ & $N$ & Series & lixact \\
\hline 15 & $3.42(2)^{a}$ & 26 & 0.9997 & 0.9997 & $3.42(2)$ & 26 & 0.9999 & 0.9999 \\
\hline 20 & $3.50(2)$ & 24 & 0.9594 & 0.9594 & $3.5](2)$ & 23 & 0.9734 & 0.9734 \\
\hline $2 j$ & $1.01(2)$ & 78 & 0.6758 & 0.6758 & $1.50(2)$ & 49 & 0.6618 & 0.6618 \\
\hline 30 & $3.10(3)$ & 239 & 0.3099 & 0.3099 & $4.50(3)$ & 147 & 0.2302 & 0.2302 \\
\hline 35 & $9.67(4)$ & 708 & 0.1121 & 0.1121 & $1.40(3)$ & 125 & 0.0526 & 0.0526 \\
\hline
\end{tabular}

1) Select a suitable value for $h$, i.e., choose $h$ so that the right-hand side of (5) is less than $\epsilon / 2$.

2) Select a suitable value for $N$, i.e., choose $N$ so that the right-hand side of (6) is less than $\epsilon / 2$.

3) Compute $P_{\text {out }}$ using these values and (4).
TABLE I

Probability of Outage, $P_{\text {out }}$

TABLE II

Probability of Outage, $P_{\text {out }}$

\begin{tabular}{cllllllll}
\hline \multirow{2}{*}{ SIR (dB) $)$} & \multicolumn{9}{c}{$m_{0}=2$} \\
\cline { 2 - 9 } & $h$ & $N$ & Series & Exact & \multicolumn{1}{c}{$h$} & $N$ & Series & Fxact \\
\hline 15 & $3.42(2)$ & 27 & 1.000 & 1.000 & $3.42(2)$ & 28 & $\mathbf{1 . 0 0 0}$ & 1.000 \\
20 & $3.51(2)$ & 23 & 0.9814 & 0.9814 & $3.51(2)$ & 23 & 0.9863 & 0.9863 \\
25 & $1.99(2)$ & 35 & 0.6548 & 0.6548 & $2.47(2)$ & 27 & 0.6509 & 0.6509 \\
30 & $5.90(3)$ & $\mathbf{1 0 4}$ & 0.1787 & 0.1787 & $7.30(3)$ & 79 & 0.1427 & 0.1427 \\
35 & $1.80(3)$ & 292 & 0.0263 & 0.0263 & $2.30(3)$ & 217 & 0.0137 & 0.0137 \\
\hline
\end{tabular}

TABLE III

Probability of Outage, $P_{\text {out }}$

\begin{tabular}{cccllclll}
\hline \multirow{2}{*}{ SIR $(\mathrm{dB})$} & \multicolumn{3}{c}{$m_{0}=5$} & \multicolumn{5}{c}{$m_{0}=5.5$} \\
\cline { 2 - 9 } & $h$ & $N$ & Serics & Fxact & $h$ & $N$ & Series & Exact \\
\hline 15 & $3.43(2)$ & 33 & 1.000 & 1.000 & $3.43(2)$ & 35 & 1.000 & 1.000 \\
20 & $3.52(2)$ & 24 & 0.9953 & 0.9953 & $3.52(2)$ & 24 & 0.9960 & 0.9960 \\
25 & $3.81(2)$ & 16 & 0.6455 & 0.6455 & $3.82(2)$ & 16 & 0.6453 & 0.6453 \\
30 & $1.33(2)$ & 35 & 0.0595 & 0.0595 & $1.44(2)$ & 31 & 0.0517 & 0.0517 \\
35 & $4.00(3)$ & 85 & $8.1817(4)$ & $8.1815(4)$ & $4.30(3)$ & 75 & $4.9797(4)$ & $4.9797(4)$ \\
\hline
\end{tabular}

These steps are very simple and can be programmed easily. Since the errors can be controlled, the method can achieve any desired precision, subject to the limits of digital computation. Therefore, only one case is treated below to exemplify the method.

Consider six mobile interferers [4] with $m_{k}=[2.4,1.8$, $4.5,0.75,1.24,3.3]$ and $\bar{p}_{k}=[1.5,1.7,3.2,2.3,3.75,2.7]$. The signal-to-interference ratio (SIR) is now defined as

$$
\text { SIR }=10 \log _{10}\left(\frac{\text { desired signal power }}{\text { average interference power }}\right) .
$$

Tables I-III show the outage probability for real and integral $m_{0}$. Entries in the "Exact" columns are computed using the exact analytical expression [4, eq. (21)]) for integral $m_{0}$ and using an adaptive Newton Cotes rule for the exact integral [4, eq. (14)]) for real $m_{0}$. The overall precision ${ }^{1} \epsilon=10^{-5}$ and the power protection ratio $q$ of $18 \mathrm{~dB}$ are used. The series solution (4) is virtually exact for both real and integral values of the fading index.

The simplicity of our method carries a price in some cases. For instance, Table I shows that the amount of computation (which depends on $N$, the series truncation point) increases as $P_{\text {out }}$ decreases (i.e., high SIR). At low $P_{\text {out }}\left(\right.$ say, $10^{-5}$ ), the value of $h$ must be small enough so that the negative domain truncation error, bounded by $\operatorname{Pr}(\gamma<-\pi / h)$, is less than $\epsilon / 2$, whereas the positive domain truncation error is negligible in this case since $\operatorname{Pr}(\gamma>0)>\operatorname{Pr}(\gamma>\pi / h)$. So unless the negative tail of the pdf of $\gamma$ decays rapidly, very small $h$ will be required, leading to large $N$. We have used the Chernoff bound to estimate $h$ : minimization of $\phi_{\gamma}(\lambda) \exp (\pi \lambda / h)$ done in the

\footnotetext{
${ }^{1}$ As pointed out by a reviewer, in cellular radio system design, one aims to guarantee $P_{\text {out }}$ of the order of $10^{-2}-10^{-3}$. Therefore, $\epsilon \approx 10^{-4}$ may be sufficient.
} 
TABLE IV

\begin{tabular}{lll} 
Probability of Outage, $P_{\text {out }}$ \\
\hline & Series & Exact [4] \\
\hline$m_{0}$ & Real & Integer \\
Derivative & No & Yes \\
Complexity & Variable & Fixed \\
\hline
\end{tabular}

range $-m_{0} q / \bar{p}_{0}<\lambda<0$. In fact, this is an unoptimized bound [i.e., $\operatorname{Pr}(\gamma<-\pi / h)<\phi_{\gamma}\left(\lambda_{1}\right) \exp \left(\pi \lambda_{1} / h\right)$ ], with $\lambda=\lambda_{1}$ being the midpoint of this interval. Increasing SIR causes this range to shrink and $\left|\lambda_{1}\right| \rightarrow 0$. That is, to ensure $\exp \left(\pi \lambda_{1} / h\right)$ is small enough, $h$ must be correspondingly small. Therefore, it may be worthwhile to estimate $\operatorname{Pr}(\gamma<-\pi / h)$ by more accurate means such as the saddle point method. However, this situation is alleviated for large $m_{0}$, which leads to larger $\left|\lambda_{1}\right|$.

Tables I-III show that for SIR of $35 \mathrm{~dB}$ and $m_{0}=(1,2,5)$, the series truncation point $N$ is 708,292 , or 85 , respectively. This shows that the number of terms to be included in the series (4) decreases as $m_{0}$ increases. Note that $f_{\gamma}(x)$ contains the exponential factor $\exp \left(x q m_{0} / \bar{p}_{0}\right)$ for $x<0$. This directly follows if $m_{0}$ is an integer because of the pole at $s=-m_{0} q / \bar{p}_{0}$. Therefore, the probability trail $\operatorname{Pr}(\gamma<-\pi / h)$ vanishes quickly for large $m_{0}$.

Table IV compares the series solution (4) and the exact solution [4]. The amount of computation for the series solution is variable, depending on $m_{0}, \epsilon$, and SIR (the variation of $N$ in Tables I-III attests to this fact). However, for the exact solution [4], higher derivatives are needed and $m_{0}$ should be an integer. A measure of the computational complexity of a numerical algorithm is expressed by the number of arithmetic operations such as additions and multiplications. An exact comparison of the computational complexity of the series solution and exact solution [4] is difficult for all integral $m_{0}$. However, for $m_{0}=1,2$, the exact solution is given by [4, eqs. (30) and (31)]. The amount of work for computing these two equations is similar to evaluating $\phi_{\gamma}(s)$ once. So in these two cases, the computational complexity of the series solution is roughly
$N$ times that of the exact solution, where $N$ is our series truncation point.

\section{CONCLUSIONS}

A characteristic function method has been presented for calculating the probability of outage in a cellular mobile radio system with Nakagami fading. This method allows all the fading severity indexes to be arbitrary real numbers in the interval $[0.5, \infty)$, requires no higher order derivatives, and can be programmed easily. More importantly, the parameters controlling the domain truncation error and the series truncation error can be selected in advance, such that the total error lies within the specified precision.

\section{REFERENCES}

[1] J.-P. Linnartz, Narrowband Land-Mobile Radio Networks. Norwood, MA: Artech House, 1993.

[2] A. Abu-Dayya and N. C. Beaulieu, "Outage probabilities of cellular mobile radio systems with multiple Nakagami interferers," IEEE Trans. Veh. Technol., vol. 40, pp. 757-767, Nov. 1991.

[3] M. Nakagami, "The $m$-distribution, a general formula of intensity distribution of rapid fading," in Statistical Methods in Radio Wave Propagation, W. G. Hoffman, Ed. Oxford, U.K.: Pergamon, 1960.

[4] Q. T. Zhang, "Outage probability of cellular mobile radio in the presence of multiple Nakagami interferers with arbitrary fading parameters," IEEE Trans. Veh. Technol., vol. 44, pp. 661-667, Aug. 1995.

[5] N. C. Beaulieu, "An infinite series for the computation of the complementary probability distribution function of a sum of independent random variables and its application to the sum of Rayleigh random variables," IEEE Trans. Commun., vol. 38, pp. 1463-1474, Sept. 1990.

C. Tellambura received the B.Sc. degree with honors from the University of Moratuwa, Sri Lanka, in 1986, the M.Sc. degree in electronics from King's College, U.K., in 1988, and the Ph.D. degree in electrical engineering from the University of Victoria, Canada, in 1993.

He was a Post-Doctoral Research Fellow at the University of Victoria from 1993 to 1994 and the University of Bradford, U.K., from 1995 to 1996. His research interests include coding, communication theory, modulation, equalization, and wireless communications. 